\title{
Aging and Fracture of Human Cortical Bone and Tooth Dentin
}

\author{
Kurt J. Koester, ${ }^{1}$ Joel W. Ager III, ${ }^{1}$ and Robert O. Ritchie ${ }^{1,2, *}$ \\ ${ }^{1}$ Materials Sciences Division, Lawrence Berkeley National Laboratory, Berkeley, CA 94720 \\ ${ }^{2}$ Department of Materials Science \& Engineering, University of California Berkeley, CA 94720
}

\begin{abstract}
Mineralized tissues, such as bone and tooth dentin, serve as structural materials in the human body and, as such, have evolved to resist fracture. In assessing their quantitative fracture resistance or toughness, it is important to distinguish between intrinsic toughening mechanisms which function ahead of the crack tip, such as plasticity in metals, and extrinsic mechanisms which function primarily behind the tip, such as crack bridging in ceramics. Bone and dentin derive their resistance to fracture principally from extrinsic toughening mechanisms which have their origins in the hierarchical microstructure of these mineralized tissues. Experimentally, quantification of these toughening mechanisms requires a crack-growth resistance approach, which can be achieved by measuring the crack-driving force, e.g., the stress intensity, as a function of crack extension ("R-curve approach"). Here this methodology is used to study of the effect of aging on the fracture properties of human cortical bone and human dentin in order to discern the microstructural origins of toughness in these materials.
\end{abstract}

Keywords: Fracture, R-curve, toughness, toughening mechanisms, bone, dentin.

\section{Introduction}

Fracture mechanics has long been used for the study of engineering materials; ${ }^{1}$ however, its utility for the characterization of mineralized tissues, such as bone and dentin, has not been as broadly realized. In its most simple application, the stress intensity ahead of a sharp stress concentrator can be evaluated using linear-elastic fracture mechanics (LEFM) and used to obtain a single-value toughness measurement, such as the $K_{I c}$ fracture toughness; in fact, this method has been used for the past several decades to evaluate the fracture resistance of bone and dentin. ${ }^{2-7}$ More recently, however, it has become apparent

\footnotetext{
* Corresponding author: tel: (510) 486-5798; fax: (510) 643-5792. E-mail address: RORitchie@lbl.gov (R. O. Ritchie)
} 
that bone and dentin principally derive their toughness during crack growth, and hence evaluation in terms of crack-growth resistance curves (R-curves) is more appropriate; indeed, several recent studies on these tissues have described their toughness properties in terms of such R-curves. ${ }^{8-11}$ There are also a few examples where nonlinear-elastic fracture mechanics (NLEFM), which allows for the presence of local plasticity (actually nonlinear elasticity), has been applied to measure the toughness of these materials; ${ }^{12-14}$ in addition, cohesive-zone modeling has been utilized as an alternative nonlinear fracture modeling approach to account for toughening and damage behavior both ahead and behind the crack

tip. ${ }^{15}$ As a critical issue is how such fracture mechanics evaluations can be related to the microstructural mechanisms of damage and toughening in human bone and teeth, we focus here on the sources of fracture resistance in these biological materials, as dictated by the characteristic size scales of their structure.

\section{Structure}

Cortical bone, the dense outer shell of bones, is a hierarchical composite of an organic phase, type-I collagen, and a mineral phase, hydroxyapatite ${ }^{16}$ it is approximately $50 \%$ mineral salts, $25 \%$ collagen, and $25 \%$ water by volume. At the nanoscale, bone is comprised of the collagen molecules and nanocrystalline hydroxyapatite; the collagen molecules self-assemble into collagen microfibrils which are impregnated with hydroxyapatite crystals. The plate-like hydroxyapatite crystals are approximately 4-6 nm x 30-60 nm x $100 \mathrm{~nm}$. The collagen microfibrils then assemble into collagen fibers which have a diameter of $\sim 1 \mu \mathrm{m}$; these in turn are arranged into the lamellar sheets which make up the cortical shell. The lamellar sheets which wrap all the way around the cortical shell are termed interstitial lamella. The process of Haversian remodeling results in concentric lamella and Haversian systems (osteons) and is the process by which intercortical bone is renewed in some adult mammals, including humans.

Haversian remodeling occurs by a cutting cone of osteoclasts resorbing bone and moving in the longitudinal direction of the bone. In the wake of the cutting cone, osteoblasts adhere to the wall of the resorbed cavity, termed the cement sheath, and begin to form new bone. The osteoblasts lay down new bone by excreting the organic extracellular matrix of bone which is subsequently mineralized. The osteoblasts become trapped in their excretions so they do not move with the cutting cone but follow via a 
process of continuous recruitment. The trapped osteoblasts mature into osteocytes, and the cavity in which they reside is called the lacuna. The lacunae are connected to each other by a network of cannaliculi. Figure 1 provides a schematic diagram of cortical bone and of the Haversian remodeling process.

Dentin is a somewhat similar mineralized tissue that comprises the bulk of the human tooth and as such determines its structural integrity. It is a hydrated composite of mineralized collagen fibers and nanocrystalline hydroxyapatite, with $\sim 45 \%$ hydroxyapatite, $35 \%$ collagen and $20 \%$ water by volume. The mineralized collagen fibrils form the intertubular dentin matrix, and are arranged in a felt-like structure oriented perpendicular to a series of channels, termed tubules. These tubules are $\sim 1-2 \mu \mathrm{m}$ in diameter, and extend from the pulp cavity to the exterior of the tooth; they are lined with a highly mineralized cuff of peritubular dentin (Fig. 2 contains a schematic of the structure of a tooth). ${ }^{17,18}$ In contrast to bone, there is no remodeling after tooth growth is completed. During aging, human dentin sclerosis causes the tubules to become occluded through deposition of carbonated apatite $^{19,20}$ leading to transparency to visual light of the dentin (termed "transparent" dentin); this leads to changes in the mechanical properties, most notably a loss in ductility, toughness and cyclic fatigue resistance. ${ }^{20-23}$

\section{Fracture Resistance}

Fracture can be considered to be a mutual competition between intrinsic damage mechanisms, which act ahead of the crack tip to promote cracking, and extrinsic shielding mechanisms, which act primarily behind the tip to inhibit cracking. ${ }^{24,25}$ Intrinsic toughening mechanisms serve to limit microstructural damage; an example is the occurrence of plasticity ahead of the crack tip, which dissipates energy and locally reduces the stresses by blunting the crack tip. This is a dominant toughening mechanism in most ductile metallic materials.

Extrinsic toughening is quite different. Unlike intrinsic mechanisms, these mechanisms do not increase the inherent resistance to fracture but instead "shield" the crack tip from the applied driving force for crack propagation; as they primarily operate in the wake of the crack tip, they require the presence of a crack and consequently result in crack-size dependent behavior. An example of an extrinsic toughening mechanism is crack bridging, where material bridges, e.g., intact fibers in fiber-reinforced composites or 
interlocking grains in monolithic ceramics, span the crack and carry load that would otherwise be used to further crack propagation (crack-tip shielding).

Bone and dentin are primarily toughened extrinsically; although intrinsic mechanisms, such as viscoplastic flow, ${ }^{26}$ have been identified, the principal source of fracture resistance arises from microcracking, ${ }^{9,11,27}$ crack deflection, ${ }^{11,13,28,29}$ and crack bridging. ${ }^{8,10,11,30,31}$ Due to the fact that extrinsic toughening mechanisms can only develop after some amount of crack extension, these mechanisms have no influence on the crack-initiation toughness (Fig. 3); rather they are associated with crack-growth toughness and naturally result in Rcurve toughness behavior where the "driving force" for cracking, e.g., $K$, $\mathcal{G}$, or $J$, increases with crack extension. Figure 3 shows a schematic diagram of an R-curve and the parameters one obtains from this type of analysis.

As noted above, a number of (extrinsic) toughening mechanisms have been identified for bone and dentin. Here, we present R-curve measurements performed on human bone and tooth dentin and discuss how such mechanisms are influenced by aging in both of these tissues. We further compare the R-curve methodology to single-value assessments of the toughness, such as $K_{I c}$.

\section{Fracture and Aging in Human Bone}

To evaluate the effect of aging on the mechanical properties of human bone, macroscopic R-curve fracture toughness tests were performed on cortical bone taken from the humerii of nine cadavers (donor age: 34 to 99 years). ${ }^{32}$ Seventeen $(N=17)$ compacttension, $\mathrm{C}(\mathrm{T})$, specimens, were fatigue precracked and tested in simulated body fluid Hanks' Balanced Salt Solution (HBSS). Samples were divided into three age groups arbitrarily named Young [age: $34(N=1), 37(N=4)$ and $41(N=2)$ years], Middle-Aged [age: $61(\mathrm{~N}=1), 69(\mathrm{~N}=2)$ and $69(\mathrm{~N}=2)$ years] and Aged [age: $85(\mathrm{~N}=1), 85(N=2)$ and $99(N=2)$ years]. The samples were all oriented with the starter notch and the nominal crack-growth direction along the proximal-distal direction of the humerus (in the longitudinal direction), i.e., parallel to the long axis of the Haversian systems and hence, long axis of the humerus. Procedures for measuring resistance-curves are detailed elsewhere. ${ }^{10,32,33}$

\footnotetext{
${ }^{a} K$ is the stress-intensity factor, $G$ is the strain-energy release rate, and $J$, the $J$-integral, is the nonlinearelastic strain-energy release rate.
} 
R-curves for cortical bone, shown in Fig. 4, give a clear indication that aging causes both the initiation toughness (intercept of the curve at zero crack extension) and the growth toughness (slope of the curve) to decrease in human cortical bone. Mean crack-initiation toughness values of 2.07 (S.D. $=0.11$ ), 1.96 (S.D. $=0.15$ ), and $1.26($ S.D. $=0.22) \mathrm{MPa} \sqrt{\mathrm{m}}$, and mean slopes (crack-growth toughness) of 0.37 (S.D. $=0.06$ ), 0.16 (S.D. $=0.01$ ), and 0.06 (S.D. $=0.04) \mathrm{MPa} \sqrt{\mathrm{m}} / \mathrm{mm}$ were obtained for the Young, Middle-Aged, and Aged groups, respectively. Differences of the means of the measured parameters (initiation toughness and growth toughness) between the groups were assessed with the one-way ANOVA statistical test and, when appropriate, one-tailed $t$ tests were used to make comparisons between the groups. These data are shown graphically in Fig. 5, where it is apparent that while aging affected both the initiation and growth toughnesses, the deterioration in properties with aging was most evident during crack growth.

With progressive aging, the bone is remodeled; in human bone this occurs in the interior of the cortex by Haversian remodeling. The process of Haversian remodeling changes the microstructure of the bone, which must be understood in terms of how this may affect, or interact with, a growing crack. Most notably, the Haversian systems have cement sheaths ("cement lines"), which have a different composition than the bulk of the bone $^{34}$ and act as weak interfaces, thus serving as sites for preferential microcracking. The process of Haversian remodeling stimulates additional remodeling due to the fact that the cement sheaths sever the canaliculi of the interstitial bone leading to the death of the osteocytes; therefore, the Haversian systems are not randomly distributed through the bone but are predominately located in the vicinity of each other. ${ }^{16}$ As expected, the density of the Haversian systems in the current study was higher in the Aged bone than in the Young bone. $^{32}$

The toughness of bone (in the longitudinal direction) deteriorates with aging due to these changes in the microstructure, which we believe inhibit the formation of uncrackedligament bridges, one of the dominant toughening mechanisms in bone. Because the cement sheaths are "weak interfaces" in bone, a crack advancing in the longitudinal direction of bone will tend to follow these features. As bone ages the density of Haversian systems increases and there is a greater chance that a crack can follow a lower resistance path along these interfaces through the material. These cement sheaths also play an 
important role in the formation of uncracked-ligament bridges. This occurs during crack propagation by a new crack initiating, generally at a cement sheath, ahead of the main crack tip; the region between the original and the new crack can then act as a bridge (the so-called "mother and daughter" crack configuration, as shown by the top left-hand image in Fig. 4), and carry load that would otherwise be used to promote cracking. This mechanism is suppressed with aging because of the higher osteon density, whereby the distance between cement sheaths is reduced, resulting in a corresponding decrease in the size of the bridges. Crack initiation can occur along a low toughness path, such as a cement sheath, and this possibly is the reason that the initiation toughness also decreases with aging. However, the principal effect of aging is on the growth toughness as the extrinsic toughening (crack bridging) mechanisms are directly affected by the age-related changes in the bone-matrix structure.

Microcracking along the cement sheaths also gives rise to toughening in the transverse (breaking) orientation of bone by providing locations for crack arrest and macroscopic crack deflections; this is shown in the x-ray computed tomography image in Fig. 6. This extrinsic mechanism of toughening is particularly effective in cortical bone and is the primary reason that bone is substantially tougher in the transverse than in the longitudinal directions.

\section{Fracture and Aging in Human Dentin}

Human molars $(N=7)$, extracted according to protocols approved by the University of California San Francisco, Committee on Human Research, were used as the source of dentin. Three-point bend samples ( $4 \mathrm{~mm}$ long, $1 \mathrm{~mm}$ wide, $0.5 \mathrm{~mm}$ thick), two or three per tooth, were wet sectioned from the central portion of the crown and root (Fig. 2) using a low speed saw, and stored in $25^{\circ} \mathrm{C} \mathrm{HBSS}$. The molars were divided into three groups, as determined by the fraction of the occluded tubules: Young dentin (19-30 years old) with 3$7 \%$ filled tubules $(N=4)$, Aged/opaque dentin (40-70 years old) with $12-32 \%$ filled tubules $(N=5)$, and Aged/transparent dentin (40-70 years old) with 65-100\% filled tubules making them transparent to visible light $(N=5)$.

R-curves for human dentin as a function of aging are shown in Fig. 7. The crackinitiation toughness is $\sim 0.75-1.1 \mathrm{MPa} \sqrt{\mathrm{m}}$ and appears to be the similar for all groups. However, after $\sim 100 \mu \mathrm{m}$ or more of crack extension, the toughness of the "young" samples 
is higher than that of the "aged" samples. This again represents an aging-related decrease in the crack-growth toughness, which can be quantified in terms of the slope (least-squares fit) of the R-curve. Results, shown in Fig. 8a, indicate that young dentin has a significantly higher $(p<0.05)$ growth toughness than aged/opaque and aged/transparent dentin; for aged dentin, the opaque group also has a significantly higher $(p<0.05)$ growth toughness than the older transparent group. Statistical differences of the means of the measured parameters (crack-growth toughness, unfilled and microcracked tubule fractions) between the groups were evaluated with the one-way ANOVA test and, when appropriate, onetailed $t$ tests were used to make comparisons between the groups. The decreased growth toughness with age is paralleled by a similar decrease in the fraction of unfilled and microcracked tubules involved in the crack path (Fig. 8).

The effect of aging in dentin is that the principal microstructural features, the tubules, become occluded with apatite mineral with age; such tubule sclerosis is thought to be the result of the in vivo loading in the mouth. ${ }^{19}$ Once the tubules have become occluded with mineral, their interaction with a propagating crack changes; specifically, the increased fraction of filled tubules in older dentin is less effective in developing extrinsic toughening mechanisms. Most notably, the unfilled tubules tend to initiate microcracks, whereas the filled tubules, presumably because they offer a reduced stress concentration, do not. Akin to bone, microcracking is important in dentin as it is a precursor to the other more potent toughening mechanisms. As cracks tend to follow a low modulus phase, the presence in young dentin of a larger fraction of empty tubules, many of which have several microcracks radiating out from them, leads to significant crack deflection and branching. Moreover, the microcracked tubules cause an increased incidence of crack bridging, particularly at larger crack sizes. This arises when a crack propagating in the intertubular dentin activates a microcrack from of a tubule which then becomes the active crack tip leaving an uncracked-ligament bridge in its wake. (This is a similar to the bridging mechanism in bone where the microcracks initiate primarily at the cement sheaths). With the progressively diminished fraction of open, and hence microcracked, tubules with aging, all these extrinsic mechanisms degrade, which results in the deterioration in the crackgrowth (but not necessarily crack-initiation) toughness of human tooth dentin with age. 


\section{Concluding Remarks}

"Hard" mineralized tissues such as human bone and tooth dentin have evolved to

effectively resist fracture. However, their enduring strength and toughness invariably degrades with age. In this brief review, we have attempted to show that in both biological materials, resistance to fracture arises primarily from crack growth (rather than crack initiation), which is evident by their rising crack-resistance curve toughening behavior. Such crack-growth toughening results from extrinsic (crack-tip shielding) mechanisms, principally associated with crack deflection and crack bridging, both processes that are induced by the formation of microcracks, which predominate at the cement sheaths in bone and at the tubules in dentin. However, with age, the increased density of Haversian systems in cortical bone and sclerosis of the tubules from mineral deposition in dentin leads to a degradation in the potency of these mechanisms, which is manifest in a reduced slope of their resistance curves.

\section{Acknowledgments}

This work was supported by the Director, Office of Science, Office of Basic Energy Sciences, Division of Materials Sciences and Engineering, Department of Energy, and by the Laboratory Directed Research and Development Program of Lawrence Berkeley National Laboratory, under contract No. DE-AC02-05CH11231.

\section{References}

1. T.L. Anderson, Fracture Mechanics Fundamentals and Applications, (Boca Raton: CRC Press LLC, 1995).

2. J.W. Melvin and F.G. Evans, Crack Propagation in Bone. in Biomechanics Symposium ASME 87-88 (New York, 1973).

3. W. Bonfield and P.K. Datta, Fracture Toughness of Compact Bone. Journal of Biomechanics 9 (1976), pp. 131-134.

4. T.M. Wright and W.C. Hayes, Fracture mechanics parameters for compact bone-Effects of density and specimen thickness. Journal of Biomechanics 10 (7) (1977), pp. 419-425.

5. J.C. Behiri and W. Bonfield, Orientation dependence of the fracture mechanics of cortical bone. Journal of Biomechanics 22 (8-9) (1989), pp. 863-867.

6. T.L. Norman et al. Mode I fracture toughness of human bone. Advances in bioengineering, Vol. 20 (ed. Vanerby) 361-364 (ASME, New York, 1991). 
7. R. De Santis et al., Bone fracture analysis on the short rod chevron-notch specimens using the X-ray computer micro-tomography. Journal of Materials Science: Materials in Medicine 11 (2000), pp. 629-636.

8. R.K. Nalla et al., Effect of orientation on the in vitro fracture toughness of dentin: the role of toughening mechanisms. Biomaterials 24 (22) (2003), pp. 3955-3968.

9. D. Vashishth, Rising crack-growth-resistance behavior in cortical bone: implications for toughness measurements. Journal of Biomechanics 37 (6) (2004), pp. 943-946.

10. R.K. Nalla et al., Mechanistic aspects of fracture and R-curve behavior in human cortical bone. Biomaterials 26 (2) (2005), pp. 217-231.

11. K.J. Koester et al., The effect of aging on crack-growth resistance and toughening mechanisms in human dentin. Biomaterials 29 (10) (2008), pp. 1318-1328.

12. P. Zioupos, Recent developments in the study of failure of solid biomaterials and bone: 'fracture' and 'pre-fracture' toughness. Materials Science and Engineering: C 6 (1) (1998), pp. 33-40.

13. H. Peterlik et al., From brittle to ductile fracture of bone. Nature Materials 5 (1) (2006), pp. 52-55.

14. J. Yan et al., How tough is bone? Application of elastic-plastic fracture mechanics to bone. Bone 40 (2) (2007), pp. 479-484.

15. Q.D. Yang et al., Fracture length scales in human cortical bone: The necessity of nonlinear fracture models. Biomaterials 27 (9) (2006), pp. 2095-2113.

16. J.D. Currey, Bones, 2nd (Princeton: Princeton University Press, 2002).

17. D.H. Pashley, Dentin: a dynamic substrate -- a review. Scanning Microscopy 3 (1) (1989), pp. 161-174.

18. A.R. Ten Cate, Oral histology-development, structure and function, (St. Louis: Mosby, 1994).

19. D.F. Weber, Human dentine sclerosis: A microradiographic survey. Archives of Oral Biology 19 (2) (1974), pp. 163-168.

20. A.E. Porter et al., A transmission electron microscopy study of mineralization in ageinduced transparent dentin. Biomaterials 26 (36) (2005), pp. 7650-7660.

21. J.H. Kinney et al., Age-related transparent root dentin: mineral concentration, crystallite size, and mechanical properties. Biomaterials 26 (16) (2005), pp. 33633376.

22. D. Arola and R.K. Reprogel, Effects of aging on the mechanical behavior of human dentin. Biomaterials 26 (18) (2005), pp. 4051-4061.

23. D. Bajaj et al., Age, dehydration and fatigue crack growth in dentin. Biomaterials 27 (11) (2006), pp. 2507-2517.

24. R.O. Ritchie, Mechanisms of fatigue crack propagation in metals, ceramics and composites: Role of crack-tip shielding. Materials science and Engineering 103 (1988), pp. 15-28. 
25. R.O. Ritchie, Mechanisms of fatigue-crack propagation in ductile and brittle solids. International Journal of Fracture 100 (1999), pp. 55-83.

26. G. Fantner et al., Sacrificial bonds and hidden length dissipate energy as mineralized fibrils separate during bone fracture. Nature Materials 4 (8) (2005), pp. 612-616.

27. D. Vashishth et al., Experimental validation of a microcracking-based toughening mechanism for cortical bone. Journal of Biomechanics 36 (1) (2003), pp. 121-124.

28. Y. Yeni and T.L. Norman, Calculation of porosity and osteonal cement line effects on the effective fracture toughness of cortical bone in longitudinal crack growth. $J$ Biomed Mater Res 51 (2000), pp. 504-509.

29. G. Parasamian and T. Norman, Diffuse damage accumulation in the fracture process zone of human cortical bone specimens and its influence on fracture toughness. Journal of Materials Science: Materials in Medicine 12 (2001), pp. 779-783.

30. Y.N. Yeni and D.P. Fyhrie, A rate-dependent microcrack-bridging model that can explain the strain rate dependency of cortical bone apparent yield strength. Journal of Biomechanics 36 (9) (2003), pp. 1343-1353.

31. R.K. Nalla et al., Mechanistic fracture criteria for the failure of human cortical bone. Nature Materials 2 (3) (2003), pp. 164-168.

32. R.K. Nalla et al., Effect of aging on the toughness of human cortical bone: evaluation by R-curves. Bone 35 (6) (2004), pp. 1240-1246.

33. E1820, Standard Test Method For Measurement of Fracture Toughness, (West Conshohocken, PA: American Society for Testing and Materials, 2006).

34. D.B. Burr et al., Composition of the cement line and its possible mechanical role as a local interface in human compact bone. Journal of Biomechanics 21 (11) (1988), pp. 939-941. 


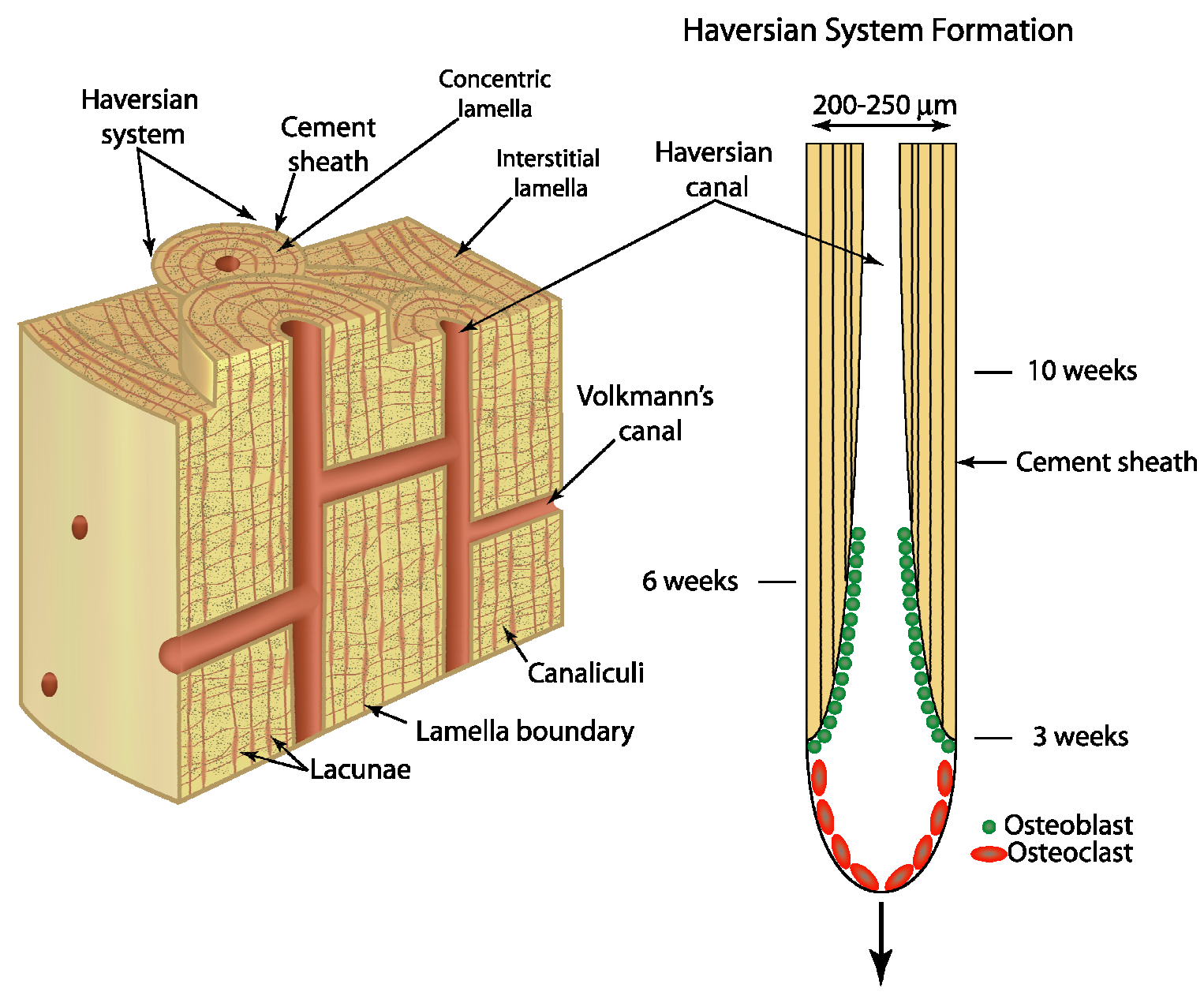

Figure 1. Diagrams of the structure of human cortical bone and of Haversian system formation. The schematic of the structure of human cortical bone shows the important microstructural features which could interact with a propagating crack. The diagram of Haversian system formation shows a cutting cone as it moves through bone and how osteoblasts follow in its wake to deposit new bone. The cutting cone advances at a rate of $\sim 40 \mu \mathrm{m}$ per day. 


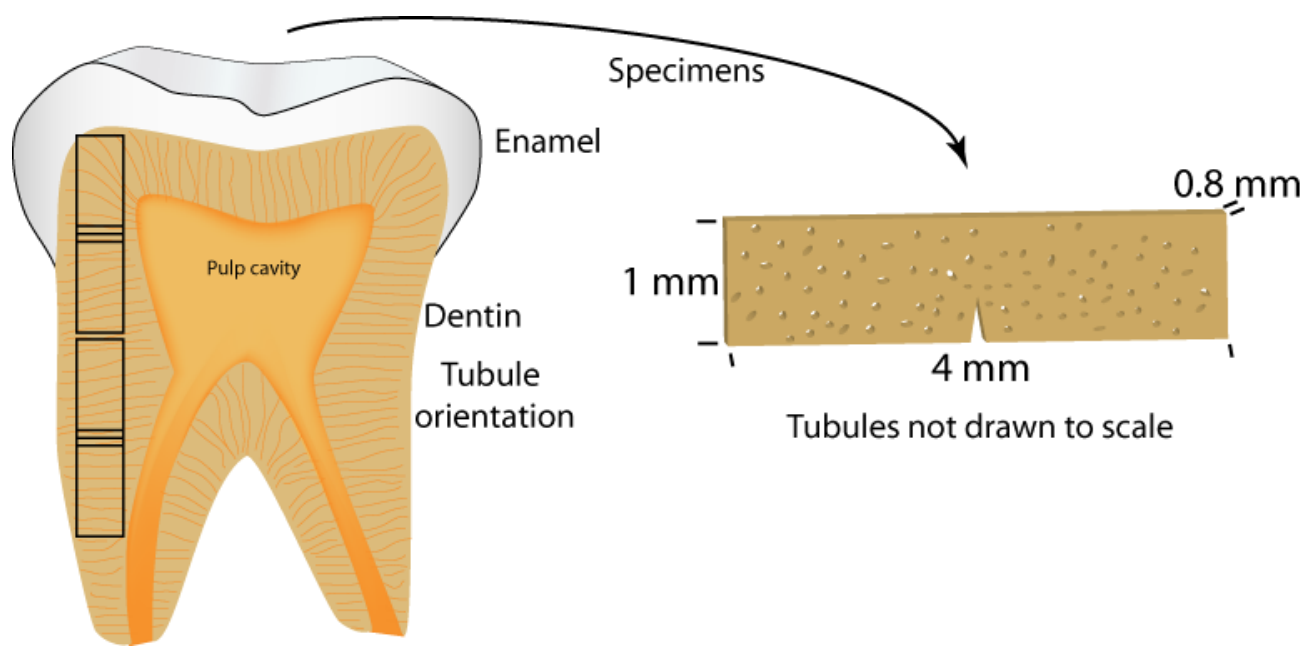

Figure 2. Schematic diagram of a human tooth (left) and of the notched three-point bend specimens of dentin (right) which were sectioned from the interior of the tooth. The nominal tubule orientation can be seen in the schematic to run from the exterior of the tooth to the pulp cavity. 


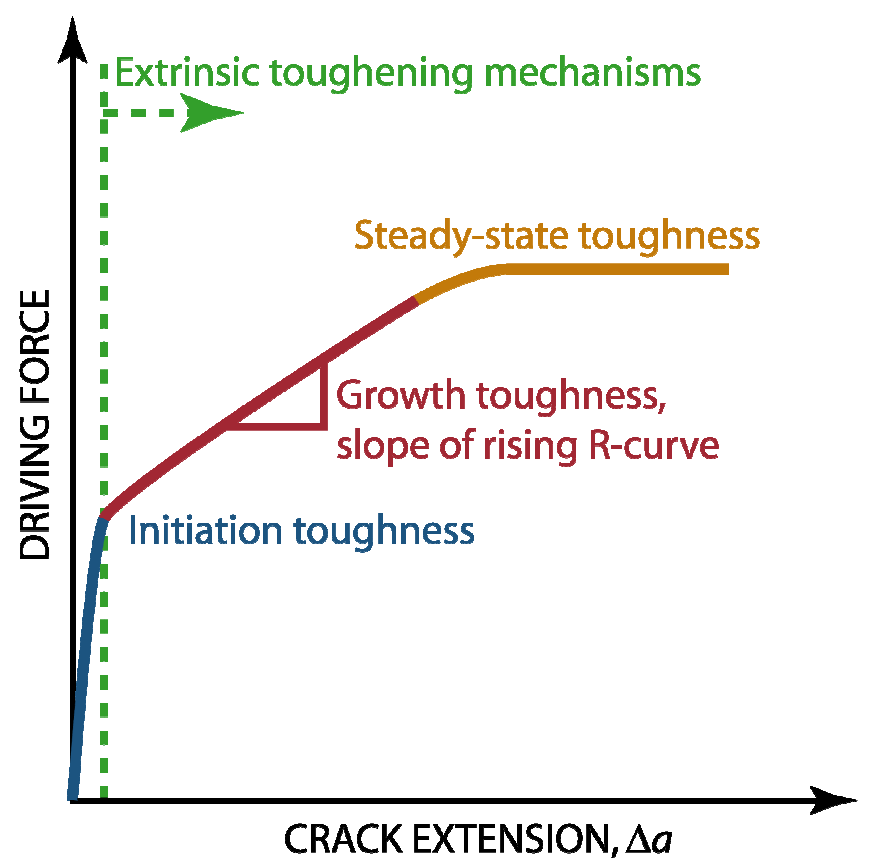

Figure 3. Schematic diagram of a crack-resistance curve (R-curve), i.e., the "crackdriving force" as a function of crack extension $\Delta a$, for a material that exhibits linearly rising R-curve behavior and in which stable cracks can be grown to sufficient lengths to measure the steady-state toughness. The crack-initiation toughness is the value of the driving force at where $\Delta a \rightarrow 0$, and in principle corresponds to values, e.g., $K_{I c}$, obtained by single-value fracture toughness parameters measurements. In the presence of extrinsic toughening mechanisms, such as crack bridging, the R-curve begins to rise with crack growth; these mechanisms require crack extension to become active. After some degree of crack extension, the toughening from these mechanisms may reach steady-state such that the toughness of the material reaches a constant value. It should be noted that in bone and dentin it is not always possible to reach steady-state due to physiological limitations on the size of the samples, as compared to the characteristic microstructural size-scales associated with the prevalent toughening mechanism. 

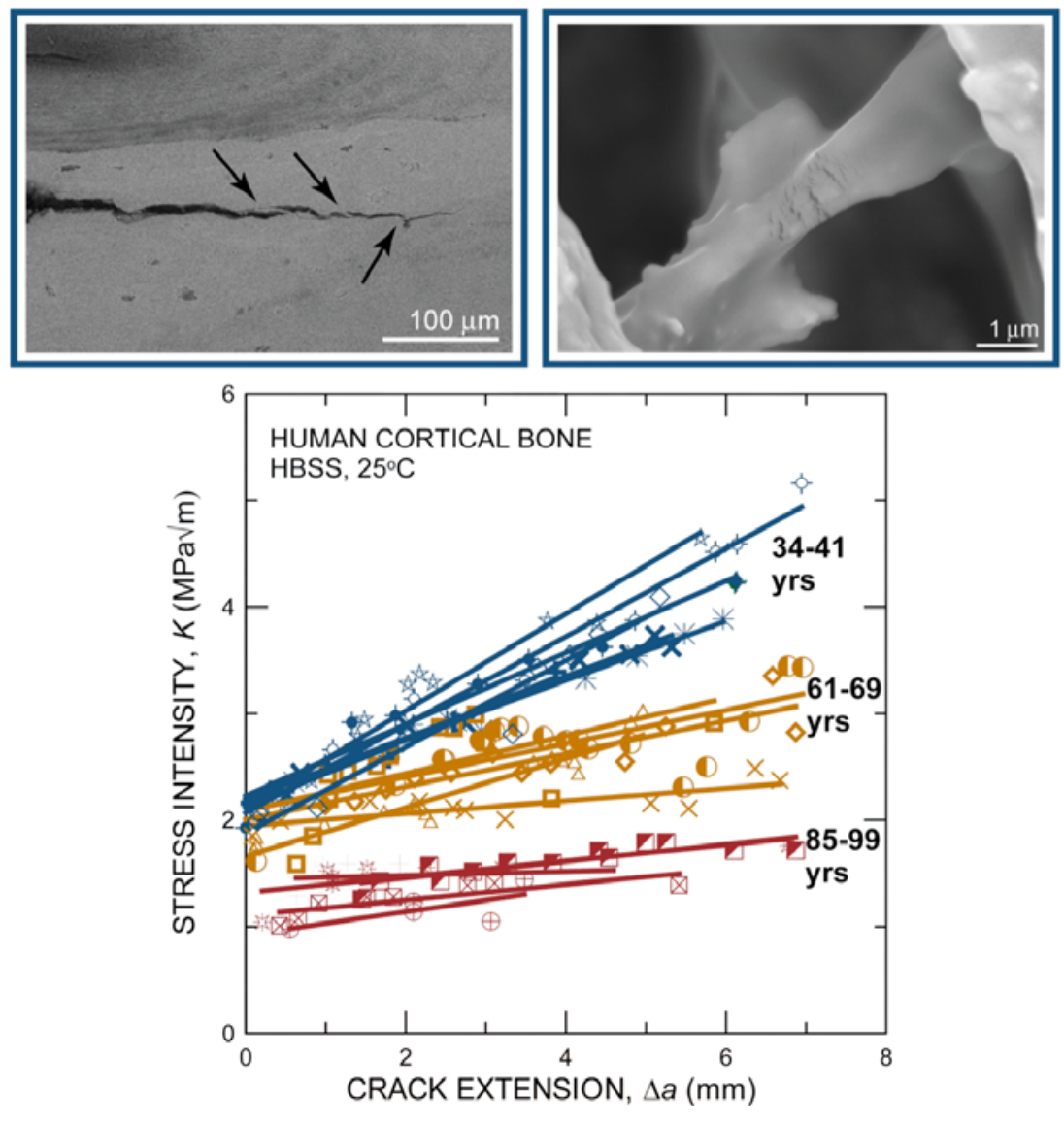

Figure 4. Crack-growth resistance curves for human cortical bone tested in $\mathrm{HBSS}$ at $25^{\circ} \mathrm{C}$, with corresponding scanning electron microscopy images of cracks in Young bone at low (left) and higher (right) magnification. The R-curves illustrate that both the initiation toughness (intercept at $\Delta a=0$ ) and the growth toughness (slope) are degraded with age. However, the more pronounced effect is on the growth toughness, which is caused by the decreasing efficacy of the extrinsic toughening mechanisms in bone with age. This in turn can be related to increased density of Haversian systems with age and the consequent reduced fraction of crack bridges in the wake of the crack. The image on the left shows the development of bridging (indicated by the arrows) for a crack propagating in the longitudinal direction; as the crack is still quite short $(\sim 200 \mu \mathrm{m})$, the bridges are still relatively small. The image on the right shows a collagen fiber bridging the crack faces. These images illustrate the dimensionality of the bridging mechanism; for example, the size-scale of the phenomenon can range from sub-micron to hundreds of microns, depending on the size of the crack and the microstructural origin of the bridging features. (adapted from ref. 32). 


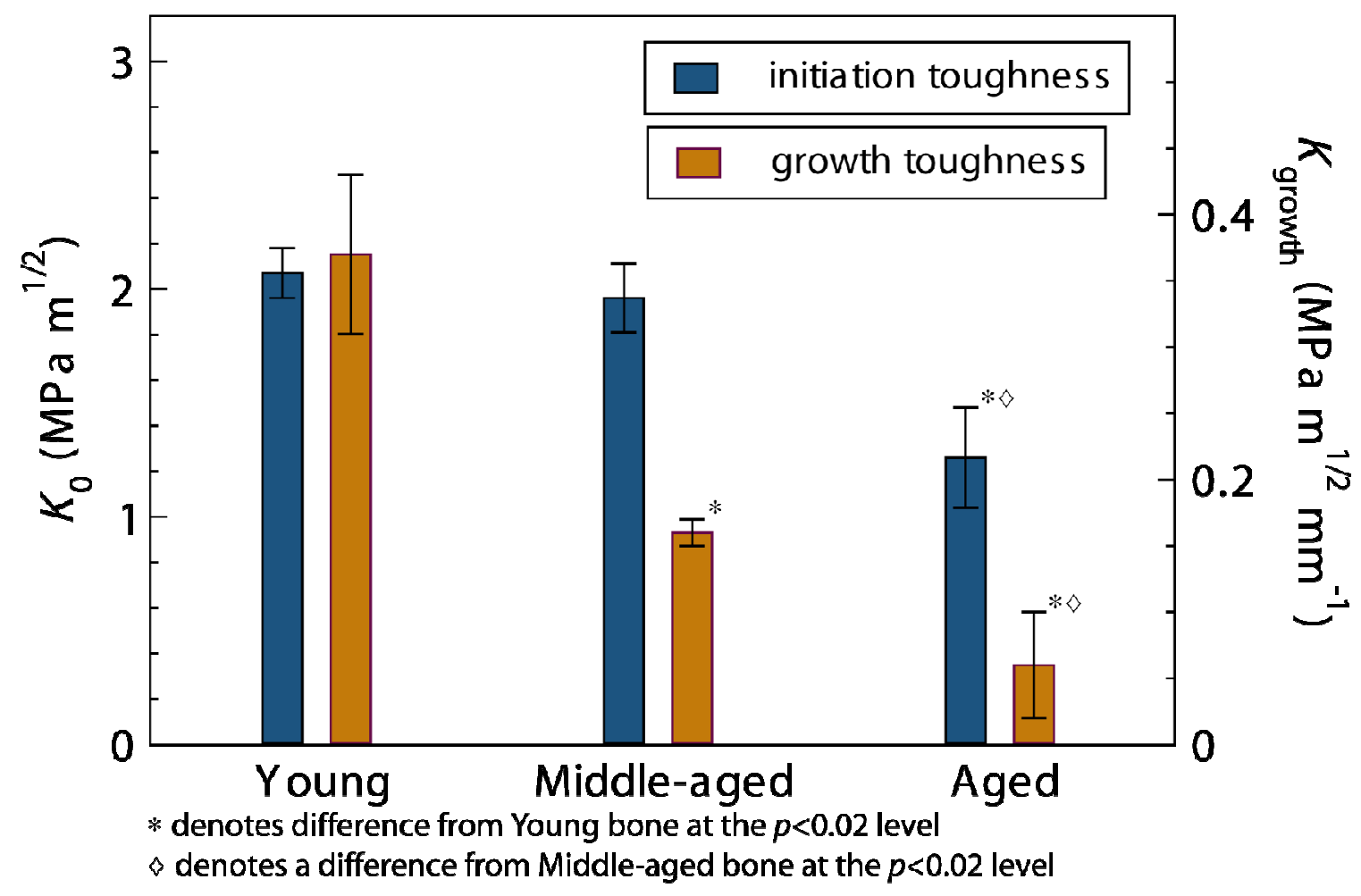

Figure 5. Means and standard deviations for the crack-initiation toughness (left-hand scale) and growth toughnesses (right-hand scale), obtained from R-curve measurements, are shown for human bone for the three groups in the study (Young = 34-41 years, Middleaged $=61-69$ years, Aged = 85-99 years). It was found using both ANOVA and $t$ tests that the deterioration in initiation toughness and growth toughness with age was statistically significant. 

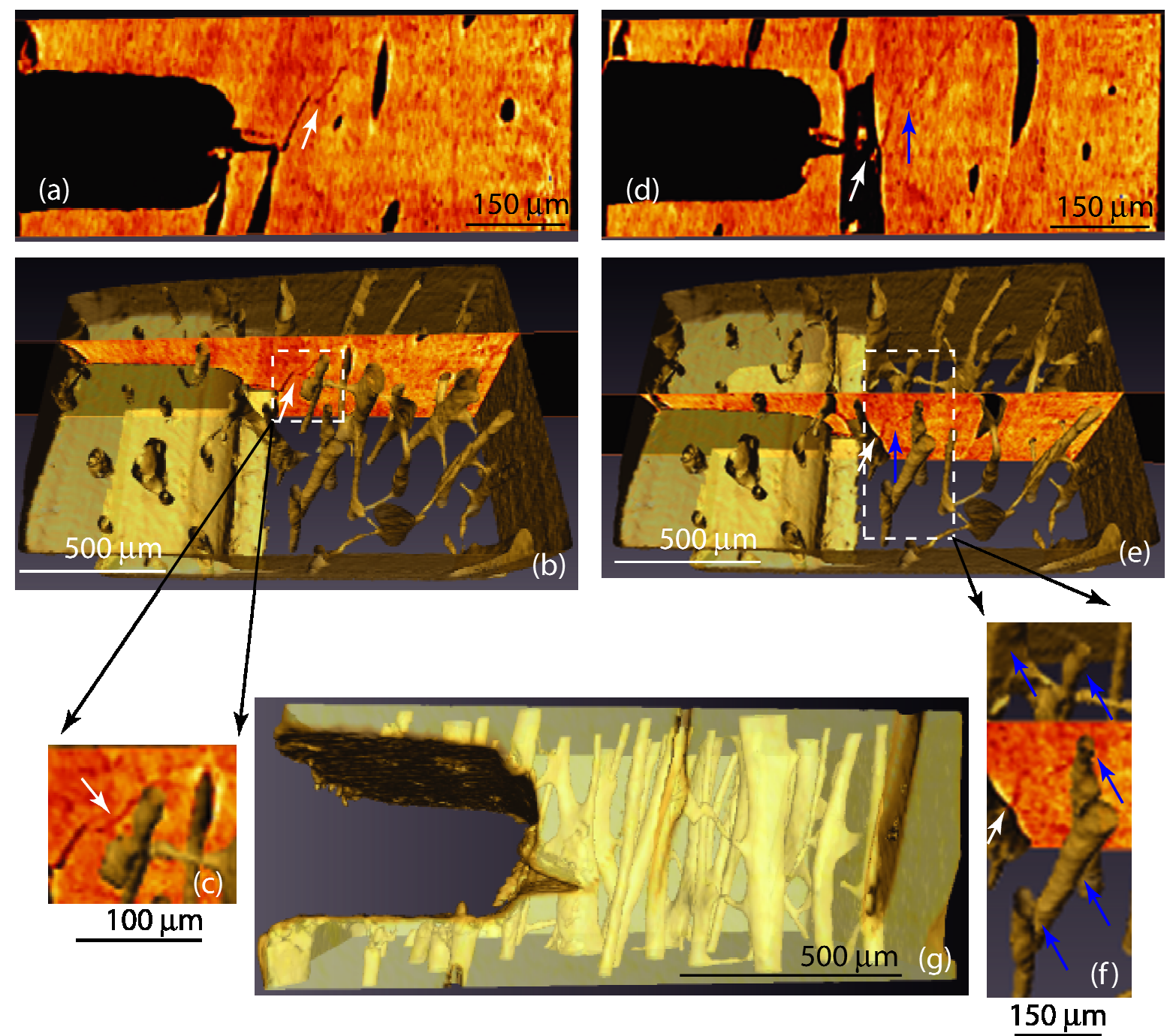

Figure 6. X-ray computed tomography images of a stable crack in human cortical bone in the transverse orientation. Panels (a-c) and (d-f) each show a two-dimensional slice through the sample, a three-dimensional image containing the slice, and a magnified view of a region of interest. A three-dimensional image of the entire volume of interest is shown in (g). In (a), a white arrow indicates a deflection that occurs before the crack encounters a Haversian canal, which appears as a black ellipse. The region of interest of this deflection is bounded by a box in (b) and the deflection is again highlighted with a white arrow. In (c) it can be seen that the deflection coincides with a Haversian canal at a different depth in the sample. Panels (d) and (e) show that at this depth the crack grows off of the notch at a different angle than the slice shown in (a), indicated by a white arrow, and that this angle corresponds to the edge of the Haversian canal at that depth. The crack can be seen to undergo three radical changes in direction in (d) and the blue arrow in (d) and (e) indicates two of these changes in direction. The region of interest in (e) given by the white box is shown in (f). In (f) the white arrow again indicates that different angle of initial crack growth and the blue arrows indicate Haversian canals that could be exerting the influence to cause the multiple deflections of the crack as seen in (d). 

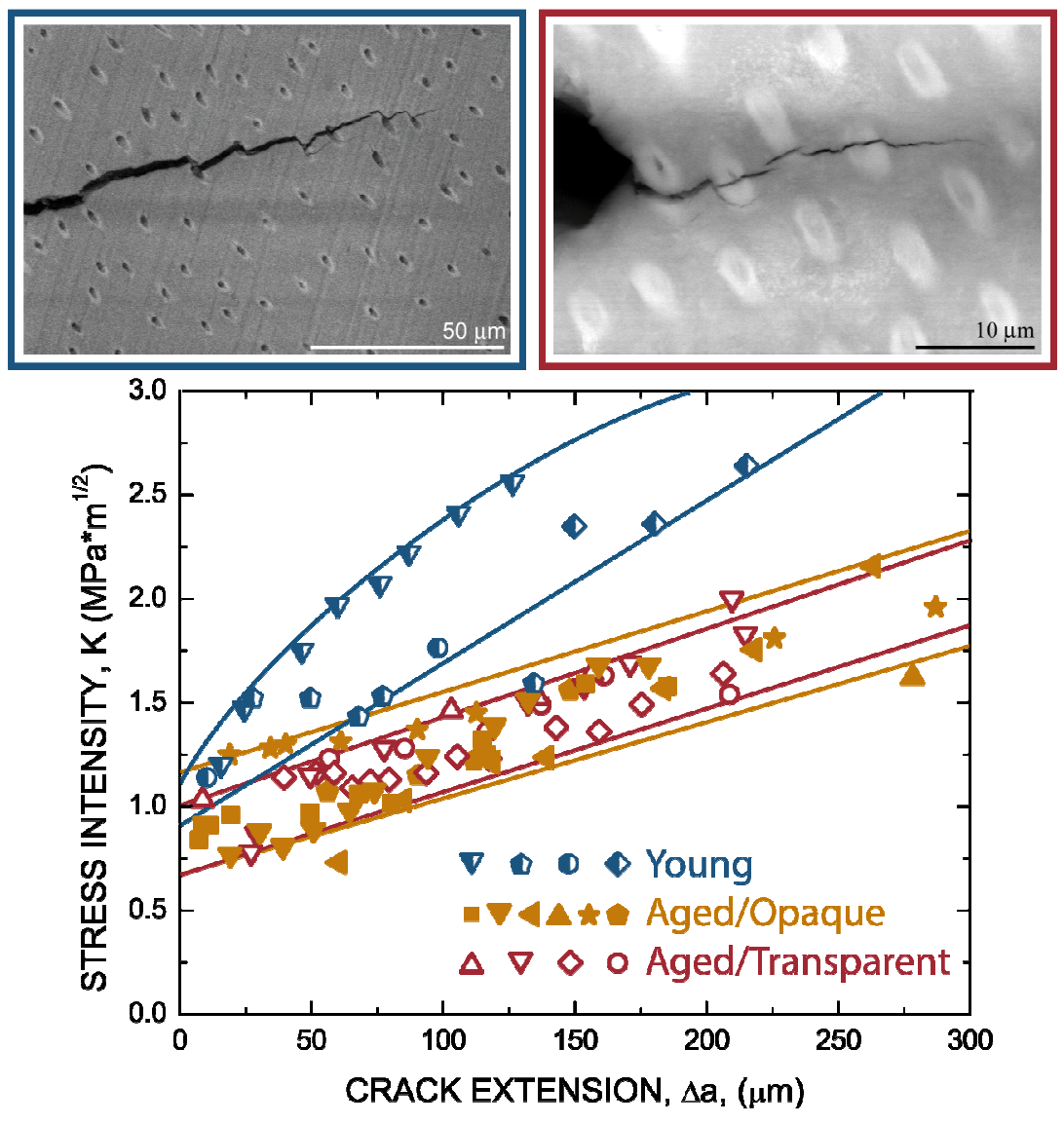

Figure 7. Crack-growth resistance curves for human tooth dentin (saturated in HBSS) grouped by age and fraction of occluded tubules. Environmental scanning electron microscopy images for the Young and Aged/transparent groups are shown in the top left and right, respectively. It can be seen from the plot that all of the groups exhibit rising Rcurve behavior. For each of the groups, the initiation toughness was difficult to determine accurately, but it was clearly similar for all groups, ranging from $\sim 0.75$ to $1.1 \mathrm{MPa} \vee \mathrm{m}$. The crack-growth toughness of the dentin, however, was found to degrade with age as the dentinal tubules became progressively occluded, which we attribute to a reduced potency of crack deflection and crack bridging toughening mechanisms (adapted from ref. 11). 

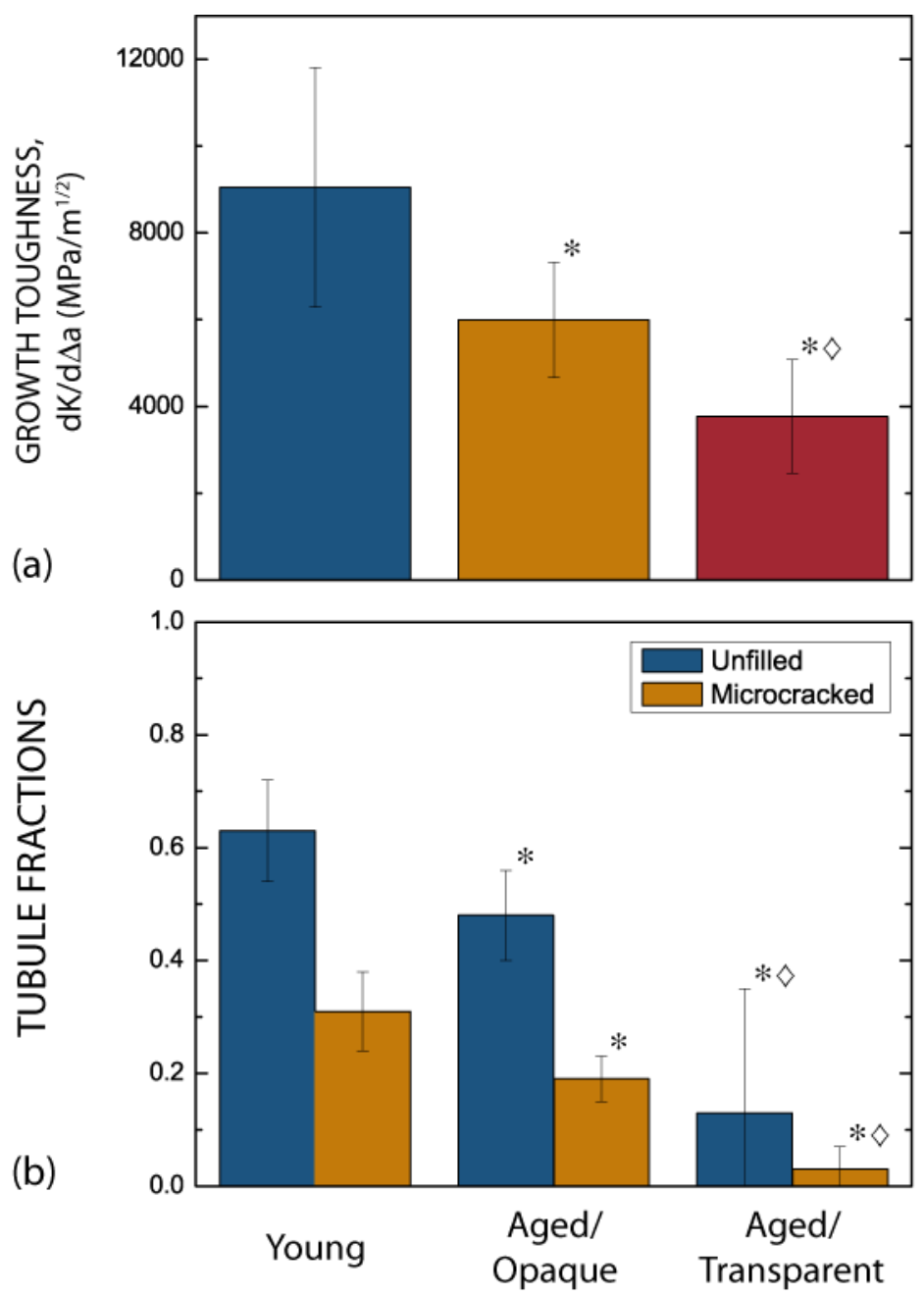

$*$ denotes difference from Young dentin at the $p<0.05$ level $\diamond$ denotes a difference from Aged/Opaque dentin at the $p<0.05$ level

Figure 8. Crack-growth toughness (a) and open tubule fractions (i.e., not occluded with mineral deposits) are shown for the Young, Aged/Opaque and Aged/Transparent human dentin groups (see text). It was found that the growth toughness degraded as the fraction of unfilled and microcracked tubules decreased. This is due to the fact that the unfilled and microcracked tubules are instrumental in the development of the extrinsic toughening mechanisms of crack deflection/branching and crack bridging; as the fraction of these tubules declined so did the occurrence and potency of these mechanisms in the crack wake. 